\title{
Catatonia and Psychosis as Manifestations of Primary Sjögren's Syndrome
}

\author{
$\underline{\text { Steeve Neves Rosado }}{ }^{1}$, Vera Silveira $^{1}$, Ana Isabel Reis ${ }^{1}$, André Gordinho ${ }^{1}$, Carla Noronha ${ }^{2}$ \\ ${ }^{1}$ Internal Medicine Residents, on Final Year - Beatriz Ângelo Hospital, Loures, Portugal \\ ${ }^{2}$ Consultant in Internal Medicine - Beatriz Ângelo Hospital, Loures, Portugal
}

Doi: 10.12890/2018_000855- European Journal of Case Reports in Internal Medicine - ( ) EFIM 2018

Received: 28/01/2018

Accepted: 05/03/2018

Published: $29 / 03 / 2018$

How to cite this article: Rosado S, Silveira V, Reis AI, Gordinho A, Noronha C. Catatonia and psychosis as manifestations of primary Sjörgen syndrome. EJCRIM 2018;5: doi:10.12890/2018_000855.

Conflicts of Interests: The Authors declare that there are no competing interests.

This article is licensed under a Commons Attribution Non-Commercial 4.0 License

\section{ABSTRACT}

Primary Sjögren's syndrome (SS) is a chronic, systemic autoimmune disease, most commonly presenting with sicca symptoms-xerostomia and xerophthalmia. Up to one-half of affected individuals also develop extra-glandular involvement. Early diagnosis is important to prevent further complications. This paper describes an atypical case of SS in a 21-year-old woman who presented first with neuropsychiatric symptoms and fever. She was diagnosed and treated for a presumed psychotic episode. However, because she remained febrile, a thorough investigation resulted in the challenging diagnosis of SS.

\section{LEARNING POINTS}

- Primary Sjögren's syndrome (SS) may be an under-recognized cause of central nervous system and neuropsychiatric involvement.

- Neuropsychiatric manifestations of primary SS can be variable.

- Patients may be treated incorrectly if the diagnosis is not rapidly established, so a clinical history and thorough examination are very important.

\section{KEYWORDS}

Sjögren's syndrome, neuropsychiatric manifestations, catatonia, psychosis

\section{INTRODUCTION}

About $10 \%$ of patients with an organic disease can present with neuropsychiatric symptoms. In some autoimmune diseases, especially systemic lupus erythematosus (SLE), neuropsychiatric symptoms are part of the diagnostic criteria. However, such manifestations are rare in other autoimmune diseases. The authors present a case of primary Sjögren's syndrome (SS) with an uncommon neuropsychiatric manifestation.

\section{CASE DESCRIPTION}

A21-year-old African female cook, without a medical history and not taking any medications, but who was using a subcutaneous contraceptive implant, was evaluated at the emergency department (ED) for behavioural disorder during the previous week. After evaluation, the patient was diagnosed with anxiety disorder and medicated with mexazolam. However, at follow-up 2 weeks later, she presented with incoherent and repetitive speech, negative symptoms and a suicide attempt with voluntary intoxication with drugs. Her family history was unremarkable. In the ED, she was haemodynamically stable, afebrile, and without notable findings on her physical examination. On neurological examination, she was calm but tense and non-compliant, her speech was monosyllabic, but she had no focal signs or other changes. Her head CT scan was 
normal, as were her laboratory results, which included thyroid function, HIV and hepatitis serology, lumbar puncture and toxicological tests. The patient was evaluated by psychiatry personnel, diagnosed as having a presumed acute psychotic episode, and admitted to the psychiatric department for investigation and management. She was started on risperidone $1 \mathrm{mg}$. She evolved to a catatonic state on the ward with negative mood, mutism, immobility, and food and drink refusal, along with fever $\left(38.7^{\circ} \mathrm{C}\right)$, and was referred to an internal medicine consultation. The fever pattern was mainly afternoon peaks (maximum temperature $39^{\circ} \mathrm{C}$ ), without any other systemic signs or symptoms. Laboratory evaluation showed isolated elevation of C-reactive protein (CRP) to $2.95 \mathrm{mg} / \mathrm{dl}$ (ref $<0.5 \mathrm{mg} / \mathrm{dl}$ ), without changes on chest X-ray or urinary tests. Blood cultures were negative and a repeated lumbar puncture was unremarkable on both cytochemical and bacteriological examination. Echocardiogram was normal. The patient remained febrile, so piperacillin and tazobactam were started empirically under the hypothesis of nosocomial respiratory infection.

Risperidone was discontinued and replaced with lorazepam $1 \mathrm{mg}$ four times a day. After initial failure to respond to medical therapy with benzodiazepines, the patient underwent electroconvulsive therapy (ECT), with progressive improvement and regression of the catatonic state after four sessions. However, she progressed to auditory and visual hallucinations and was started on low-dose clozapine, with progressive increase to $125 \mathrm{mg}$, with regression of hallucinations. However, after 7 days of antibiotics, the fever still persisted. Examination revealed bilateral inguinal and left axillary microadenopathies, new onset bicytopenia, with normocytic anaemia (haemoglobin $11.7 \mathrm{~g} / \mathrm{dl}$;

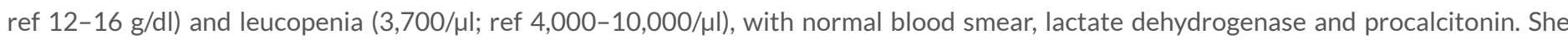
also had slightly elevated CRP (1.19 mg/dl) and an erythrocyte sedimentation rate (ESR) of $87 \mathrm{~mm} / 1 \mathrm{st}$ hour (ref $<25 \mathrm{~mm} / 1 \mathrm{st} \mathrm{hour).} \mathrm{Her} \mathrm{full-}$ body scan revealed multiple adenopathies in axillary, inguinal, iliac and lumbo-aortic territories, as well as ground-glass opacity on the right superior lobe, with a normal bronchofibroscopy. The laboratory evaluation included an autoimmune panel that was positive for ANA, titre 1/640 (ref <1/160), positive for anti-Ro/SSA and La/SSB, weakly positive for IgM for anticardiolipin and anti-B2-glicoproteín 1 with negative IgG, negative for anti-dsDNA, and showed normal complement and polyclonal hypergammaglobulinemia with elevated lgG (2120 mg/dl; ref $670-1,560 \mathrm{mg} / \mathrm{dl}$ ). The cranial angio-MRI was normal and the histological exam from inguinal adenopathy excision showed reactive benign adenopathy.

At that time the patient had no psychotic symptoms but had confirmed prior sicca symptoms. Because of the strong hypothesis of autoimmune disease (SLE or SS, with neurological involvement), antipsychotics were discontinued and she was started on methylprednisolone $1 \mathrm{~g} / \mathrm{daily}$ for 3 days, followed by prednisolone $1 \mathrm{mg} / \mathrm{kg} /$ day, in association with hydroxychloroquine $400 \mathrm{mg} / \mathrm{day}$ and acetylsalicylic acid (ASA) $75 \mathrm{mg} /$ day, with clinical (remission of fever and return to normal psychological state) and laboratory improvement, sustained after withdrawal of anti-psychotic therapy. She also underwent a salivary gland biopsy that revealed a lymphocytic infiltrate (Chisholm-Mason grade III), while the ophthalmological examination, performed after complaints of mild sicca symptoms, revealed keratitis. She was discharged from hospital on hydroxychloroquine, ASA and prednisolone, gradually tapered until discontinuation. She improved and remains asymptomatic after 1 year of follow-up.

\section{DISCUSSION}

SS, more common after the fourth decade of life ${ }^{[1]}$, is one of the most common connective tissue disorders with a 9:1 ratio between women and men ${ }^{[2]}$. Characterized by lymphocytic infiltration of exocrine glands, causing the traditional sicca symptoms of oral and ocular dryness, it can be primary or secondary to other diseases, mostly SLE and rheumatoid arthritis ${ }^{[1]}$. The presence of positive anti-Ro/SSA and La/SSB is the hallmark of the disease, with the latter being highly specific ${ }^{[2]}$.

Extraglandular manifestations may be present, with involvement of the central nervous system (CNS) being reported in $10-70 \%$ of cases ${ }^{[3]}$. Mild to moderate cognitive or psychiatric impairment can be present in up to $80 \%$ of patients ${ }^{[4]}$. Presentation is variable, with difficulties in diagnosis due to the presence of other early manifestations in many cases ${ }^{[3]}$. The association with psychiatric symptoms is an example of CNS involvement, presenting most commonly as mood disorders, but also as dementia, psychosis, schizophrenia or other personality disorders, which have all been described ${ }^{[1,4,5]}$.

This case represents a rare manifestation of primary SS with a catatonic state and psychosis. To the best of our knowledge, no similar cases have been described previously in the literature. Early anamnesis was difficult in our patient due to her presentation and diagnosis was based, initially, on physical examination and laboratory findings, supported by the autoimmune panel and response to therapy. The diagnosis was later confirmed by the presence of lymphocytic infiltrate in salivary glands and keratitis. The patient was treated with corticosteroids and hydroxycloroquine, with immediate clinical improvement, and was able to stop steroids. The decision that no further immunosuppressive or anti-psychotic treatment was necessary was based on the excellent response and no recurrences during 1-year follow-up. 


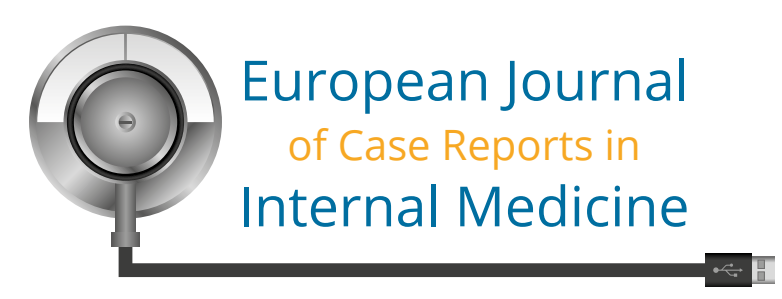

\section{CONCLUSION}

Primary SS may affect the CNS in a large number of patients. As described here, unexplained neuropsychiatric manifestations can be the first sign of the disease, so SS should be part of the differential diagnosis in patients with these new-onset symptoms, so that the diagnosis is not overlooked.

\section{REFERENCES}

1. Lin CE. One patient with Sjögren's syndrome presenting schizophrenia-like symptoms. Neuropsychiatr Dis Treat 2016;12:661-663.

2. Mavragani CP, Moutsopoulos HM. Sjögren syndrome. CMAJ 2014;186:E579-586.

3. Delalande S, De Seze J, Ferriby D, Vermersch P. Neurological manifestations in Sjögren syndrome. Rev Med Interne 2010;31(Suppl 1):S8-15.

4. Cox PD, Hales RE. CNS Sjögren's syndrome: an underrecognized and underappreciated neuropsychiatric disorder. J Neuropsychiatry Clin Neurosci 1999;11:241-247.

5. Pelizza L, Bonacini F, Ferrari A. Psychiatric disorder as clinical presentation of primary Sjögren's syndrome: two case reports. Ann Gen Psychiatry $2010 ; 9: 12$. 